of experience and confidence in DA enquiry and disclosure management improved dramatically over this period.

\section{P145 AUSTRALIAN MSM'S VIEWS AND KNOWLEDGE OF PHARYNGEAL GONORRHOEA, WILLINGNESS TO CHANGE CURRENT SEXUAL PRACTICES AND THE ACCEPTABILITY OF USING MOUTHWASH TO REDUCE THE RISK OF PHARYNGEAL GONORRHOEA: A QUALITATIVE STUDY}

\begin{abstract}
${ }^{1,2}$ sandra Walker ${ }^{*}{ }^{1,2}$ Clare Bellhouse, ${ }^{1,2}$ Jade Bilardi, ${ }^{1,2}$ Christopher Fairley, ${ }^{1,2}$ Eric Chow. ${ }^{1}$ Melbourne Sexual Health Centre, melbourne, Australia; ${ }^{2}$ Monash University, melbourne, Australia
\end{abstract}

\subsection{6/sextrans-2016-052718.199}

Background/introduction The pharynx is the most common site of gonorrhoea among men who have sex with men (MSM) and may serve as a reservoir for infection, with saliva implicated in transmission possibly through oral sex, kissing, and rimming. Reducing sexual activities involving saliva may reduce pharyngeal gonorrhoea however strategies that target the oral cavity warrant investigation.

Aim(s)/objectives This study aimed to explore MSM's views and knowledge of pharyngeal gonorrhoea, their willingness to change saliva transmitting sexual practices and the acceptability of using mouthwash to reduce transmission.

Methods 30 MSM, recruited from a sexual health clinic in Melbourne, Australia, were interviewed face to face or by telephone. Results Most men considered pharyngeal gonorrhoea non-serious and attributed transmission to saliva and oral ejaculate. Most men would not stop kissing $(n=25)$, oral sex $(n=26)$, or consider using condoms for oral sex $(n=25)$ to reduce their risk of gonorrhoea. Kissing and oral sex were common and considered enjoyable but regarded as low risk sexual activities. Men were more likely to consider stopping sexual activities they did not enjoy or practice often including rimming $(\mathrm{n}=21)$ and using saliva as a lubricant for anal sex $(n=28)$. If proven effective, most men reported they would use a mouthwash to reduce or prevent their risk of pharyngeal gonorrhoea.

Discussion/conclusion MSM are unlikely to stop sexual practices they enjoy and consider low risk. The findings from this study highlight the need for further exploration of innovative strategies such as mouthwash to reduce their risk of pharyngeal gonorrhoea.

\section{P146 DIAGNOSING GONORRHOEA - HOW DO DOCTORS AND NURSES COMPARE? AN AUDIT OF GONORRHOEA MANAGEMENT IN A LARGE PROVINCIAL NHS TRUST}

${ }^{1}$ Harriet Eatwell*, ${ }^{1}$ Qiang Lu, ${ }^{2}$ Elizabeth Foley, ${ }^{1,2}$ Rajul Patel. ${ }^{1}$ University of Southampton, Southampton, UK; ${ }^{2}$ Solent NHS Trust, Southampton, UK

\subsection{6/sextrans-2016-052718.200}

Background/introduction Although gonorrhoea rates are rising, incidence of urethral and cervical infection remain low in comparison to historic data. There is therefore concern that expertise in microscopic diagnosis of gonorrhoea may be falling. Additionally, in light of emerging resistance of gonorrhoea to extendedspectrum cephalosporins, multiple guidelines highlight the importance of taking cultures from NAAT positive sites prior to antibiotic treatment.
Aim(s)/objectives To evaluate the sensitivity of urethral microscopy performed by doctors/nurses and the frequency with which cultures are taken from all NAAT positive sites prior to treatment.

Methods A retrospective case note review of 100 patients with a gonorrhoea diagnosis and all gonorrhoea contacts in the same time period.

Results 16 men with genitourinary symptoms had positive urethral cultures on initial visit. 16/16 (100\%) had positive microscopy. 32 men with genitourinary symptoms had a positive urethral/urine NAAT, of which 30 had microscopy. In 25/30 (83\%), microscopy was positive. When performed by doctors, this was $7 / 8(88 \%)$, and by nurses was $18 / 22(82 \%)(\mathrm{p}=0.46)$. 64 patients with a positive NAAT were consulted by exclusively doctors or nurses before treatment. 11/15 (73\%) of doctors' patients and 30/49 (61\%) of nurses' patients had cultures taken from all NAAT positive sites before treatment $(\mathrm{p}=0.12)$.

Discussion/conclusion Microscopy in men with genitourinary symptoms remains sensitive in comparison with culture. However, there may be a case for a new auditable standard comparing microscopy with NAATs. Doctors and nurses are inconsistent in taking cultures from all NAAT positive sites prior to treatment and training in both groups should be addressed.

\section{P147 ADDING A DOMESTIC ABUSE ROUTINE PROMPT TO THE GUM PROFORMA: BUT ARE WE ASKING THE QUESTION?}

Rachel Sacks*, Anthi Lavida, Alison Mears. Imperial College Healthcare NHS Trust, London UK

\subsection{6/sextrans-2016-052718.201}

Background/introduction In July 2015, a routine domestic abuse (DA) prompt was introduced in a busy, walk-in, inner-London, genitourinary medicine (GUM) clinic. DA guidelines, proforma and management flowchart were devised. Tiered training was/is provided at a basic level for all staff and in-depth for Sexual Health Information Protection team (SHIP) and DA champions. Auditable outcomes: DA question asked where safe (Target 100\%), (SAFE: quiet/confidential space, seen alone, no child $>18$ months present, professional interpreter if necessary), Complete DA proforma if DA disclosed (100\%), Patient information leaflet (PIL) given if DA > 3/12 ago/no on-going risk (100\%), Offered SHIP referral for risk assessment if DA $<3 / 12$ or on-going risk (100\%), DA disclosures correctly coded $(100 \%)$.

Aim(s)/objectives Audit whether DA routine prompt asked, proforma completed, initial management pathway followed and disclosures coded.

Methods Data collected (notes review) on 100 consecutive, new, walk-in, GUM patients > 18 years-old, from $1^{\text {st }}$ October 2015.

Results 59 female, 41 male. $91 \%$ patients asked about DA. 9 not asked: 5/41 (12.1\%) male, 4/59 (6.8\%) female. 9/9: no reason documented explaining omission. 5/91 (5\%) disclosed DA (all female). DA proforma completed in $3 / 5$ (60\%) (1 patient declined further discussion). 1/4 (25\%) had current/on-going risk and referred to SHIP. 3/4 DA occurred $>3 / 12$ ago/no on-going risk: 1 accepted, 1 declined, 1 not offered PIL. 2/5 (40\%) DA disclosures coded correctly (using in-house code).

Discussion/conclusion High enquiry rate (male patients less likely to be asked). DA protocol/flow chart followed in the 